\title{
Magnetic Resonance Imaging Features of Idiopathic Granulomatous Mastitis: A Retrospective Analysis
}

\author{
Necdet Poyraz, ${ }^{1,}$ Ganime Dilek Emlik, ${ }^{2}$ Abdussamet Batur, ${ }^{2}$ Ebubekir Gundes, ${ }^{3}$ and Suat Keskin ${ }^{1}$ \\ ${ }^{1}$ Department of Radiology, Meram School of Medicine, Necmettin Erbakan University, Konya, Turkey \\ ${ }^{2}$ Department of Radiology, School of Medicine, Yuzuncu Yil University, Van, Turkey \\ ${ }^{3}$ Department of General Surgery, Agri State Hospital, Agri, Turkey \\ "Corresponding author: Necdet Poyraz, Department of Radiology, Meram School of Medicine, Necmettin Erbakan University, Konya, Turkey. Tel: +90-5334931774, Fax: \\ +90-3322237967, E-mail: necdetpoyraz@gmail.com
}

Received 2014 June 04; Revised 2014 November 14; Accepted 2014 November 24.

\begin{abstract}
Background: Granulomatous mastitis is a rare and benign inflammatory breast disease that may clinically and radiologically mimic breast cancer.

Objectives: The aim of this study was to evaluate the features of idiopathic granulomatous mastitis (IGM) on breast magnetic resonance imaging (MRI) with mammographic and sonographic findings.

Patients and Methods: A retrospective analysis was conducted on 20 patients with IGM who had been diagnosed by needle core or excisional biopsy at a single institution between 2006 and 2012. All of the patients underwent MRI for suspicious breast findings prior to biopsy. MRI examinations were performed on a $1.5 \mathrm{~T}$ scanner. The MRI findings were evaluated in accordance with the breast imaging-reporting and data system (BI-RADS) MRI lexicon established by the American College of Radiology.

Results: MRI detected a total of 29 lesions in the 20 patients. Fourteen of these lesions were seen as mass enhancements, with the remaining 15 identified as non-mass enhancements (NMEs). The median size of all lesions was $3.6 \mathrm{~cm}$ (range, $0.7-6.7 \mathrm{~cm}$ ). The most frequently observed features were masses with a round shape ( 9 out of $14,64 \%$ ), smooth contour (11 out of 14, 78\%), and a rim enhancement pattern (10 out of 14, 71\%). The most common features of the 15 NME lesions were segmental distribution (6 out of 15 , $40 \%$ ) and heterogeneous enhancement patterns ( 8 out of $15,53 \%$ ). The time-intensity curves of the dynamic studies showed benign type one signal intensity (persistent enhancement pattern) in the majority of lesions (10 out of 20,50\%).

Conclusion: Our study suggests that breast MRI findings of IGM have a wide spectrum. Rim enhancement patterns are frequently seen on contrast enhanced images, but the imaging findings are nonspecific and cannot be used definitively to distinguish between benign and malignant lesions.
\end{abstract}

Keywords: Breast MRI, Idiopathic Granulomatous Mastitis, BI-RADS

\section{Background}

Granulomatous mastitis is a rare and benign inflammatory breast disease. It is classified into two groups: specific (tuberculosis, sarcoidosis, foreign body, wegener's granulomatosis, and fungal and parasitic infections) and idiopathic. Idiopathic granulomatous mastitis (IGM) is histopathologically diagnosed by detecting non-caseified granulomatous lobulitis and excluding other factors. In general, it initially manifests clinically as a painful mass in the breast but later develops into a skin ulcer, abscess, or fistula. Although it is a benign condition, it may clinically and radiologically mimic breast cancer (1-3).

\section{Objectives}

To our knowledge, mammographic and sonographic findings of IGM have been reported previously, but magnetic resonance imaging (MRI) examinations of patients with IGM are limited in the literature (4-7). The aim of this study was to evaluate the MRI findings of IGM in particular, and to investigate the usefulness of MRI in distinguishing IGM from breast carcinoma.

\section{Patients and Methods}

In this study, we conducted a retrospective review of the pathological reports of patients with mastitis at our institution between June 2006 and February 2012. The institutional research ethics committee approved this retrospective study. 
Patients who had clinically and histologically proven simple and other specific granulomatous mastitis (sarcoidosis, tuberculosis, etc.) were excluded from the study. In total, 35 patients with IGM were identified. The clinical findings were obtained from a retrospective review of the patients' records. Of the 35 patients with IGM, 20 had undergone MRI examinations prior to Tru-cut biopsy or excisional biopsy procedures, and were thus included in this study.

Histologic analysis was based on the analysis of hematoxylin and eosin-stained slides. The identification of non-caseating granulomas in a lobulocentric distribution was compatible with the diagnosis of IGM. Epithelioid macrophages were often identified, as well as multinucleated giant cells. All slides with polarized light excluded a foreign body granulomatous reaction. There was no evidence of vasculitis, tuberculosis, or sarcoidosis, clinically or histologically.

Mammography examinations were performed on the women who were older than 30-years-old. Eleven of the 20 patients' records were available for review. Standard craniocaudal and mediolateral oblique views were obtained in all cases, with additional views when required. The ultrasonographic results of the 20 patients were available for review. Ultrasonography was performed using a 7-10 $\mathrm{MHz}$ linear transducer in our breast center.

\subsection{MR Protocol}

MRI was performed on all patients using a $1.5 \mathrm{~T}$ scanner(Magnetom Avanto; Siemens, Erlangen, Germany) with a dedicated breast coil. The scanning protocol included axial T2-weighted fat-suppressed spin-echo sequences and pre-contrast axial T1-weighted spin-echo sequences, followed by an axial pre-contrast and post-contrast three dimensional turbo fast low-angle shot (3D Turbo FLASH) sequence. Gadopentetate dimeglumine was administered through the antecubital vein at a dose of $0.1 \mathrm{mmol} / \mathrm{kg}$ of body weight. Dynamic contrast-enhanced image acquisition was commenced immediately after the contrast injection. For post-contrast imaging, five 3D Turbo FLASH sequences were repeated without time gaps, and each of the obtained images were subtracted from the pre-contrast images.

\subsection{Interpretation of MR Findings}

The assessments were conducted by two radiologists (DE and NP), and correlated with the mammography and ultrasonography results at a workstation (Leonardo; Siemens, Erlangen, Germany). The MRI findings of the lesions were defined according to the breast imagingreporting and data system(BI-RADS) lexicon established by the American college of radiology (3). After identifying the lesions as masses or non-mass enhancements (NMEs), their morphological features were analyzed in terms of their enhancement patterns and kinetic curves. The masses were evaluated according to their shapes (round, oval or irregular), margins (smooth, irregular or spiculated), and internal enhancement features (homogeneous, heterogeneous, or rim enhancement). The NME characteristics were also evaluated according to their distribution (clumped, linear, segmental, regional, or diffuse) and internal enhancement patterns (homogeneous, heterogeneous, clumped, or reticular). The T2-weighted signal intensity on the non-contrast images was recorded for all of the lesions. The time-signal intensity curves (persistent, plateau or washout) were set for all of the lesions. The contrast kinetic curves were assessed according to three types: a persistent contrast pattern with a progressive increase(type1), a plateau pattern (type 2 ), and a washout contrast pattern (type 3).

According to the morphologic analysis, the presence of a focal mass with a round, oval shape and a smooth margin, as well as unilateral, asymmetric NMEs in a regional distribution, were identified as negative findings for malignancy. However, the presence of masses with irregular or spiculated margins, heterogeneous or rim enhancement, and NMEs, especially of clumped or linear types in ductal or segmental distributions, were defined as positive findings. According to the kinetic analysis, a type 1 kinetic curve was defined as a negative finding. On the other hand, type 2 and type 3 were defined as positive findings for malignancy. Finally, imaging findings were attributed to one of the six BI-RADS categories. Following imaging, a guided core biopsy was performed in all cases for confirmation of the diagnosis.

\section{Results}

All 20 patients were women, and the mean age was 38 years at the time of presentation (range 25 to 58 years). Fourteen patients presented at the screening examination with a palpable breast mass accompanied by erythematous skin changes, with the remaining six each having a palpable mass without associated inflammatory signs. Of 20 patients, 14 were given antibiotics, but no patients had received steroid therapy before the MRI examination. None of the women had any systemic disorders or a history of specific infections. The right breast was involved in six (30\%) of the patients and the left breast in 14 (70\%).

The most common mammographic findings included asymmetric densities with no distinct margins in seven patients (BI-RADS 0), circumscribed masses in three (BI-RADS 
3), and an ill-defined mass in one (BI-RADS 4). Microcalcifications were not seen in any of the patients. Ultrasonography showed irregular heterogeneous hypoechoic lesions with or without tubular extension in 10 patients (BI-RADS 4), complex cysts containing thick walls and septa with fistula formation in four patients (BI-RADS 3), and parenchymal heterogeneity and subcutaneous fat obliteration in six patients (BI-RADS 0).

In total, MRI identified 29 primary lesions in the 20 patients. Of these 29 lesions, 14 (48\%) were seen as mass enhancements, while 15 (52\%) were considered NMEs (Figure 1). Both NMEs and mass lesions were seen in nine patients (9 out of 20,45\%). NME lesions alone were detected in six patients ( 6 out of $20,30 \%$ ), and mass enhancements alone were found in five patients ( 5 out of $20,25 \%$ ).

The most frequently observed features were masses with a round shape ( 9 out of 14, 64\%), a smooth contour (11 out of $14,78 \%$ ), and a rim enhancement pattern (10 out of 14 , $71 \%)$. Of the 15 NMEs, the most common features were segmental distribution ( 6 out of $15,40 \%$ ) and heterogeneous enhancement patterns (8 out of 15, 53\%) (Figures 2 and 3). The results are summarized in Table 1.

The time-intensity curves of the dynamic studies showed benign type 1 kinetic curves (persistent enhancement pattern) in the majority of lesions (16 out of 29 , $55 \%$ ). Type 2 (plateau enhancement pattern) was detected in nine lesions, while malignant type 3 (washout pattern) was found in four lesions (Figure 4). MRI detected ring-like enhanced masses with smooth margins within an area of segmental NMEs in three of four lesions with type 3 kinetic curves. While asymmetric density was seen on mammography, ultrasonography showed hypoechoic lesions with tubular extension in these three patients. On MRI, another patient had an irregular homogeneous enhancement lesion. This lesion was demonstrated as an ill-defined and irregular hypoechoic mass on mammography and ultrasonography, respectively. Fourteen mass lesions on MRI could be detected on ultrasonography, but only four lesions were seen on mammography. Fifteen NME lesions seen on MRI, with or without masses, were detected on ultrasonography in 10 lesions as diffuse and local parenchymal heterogeneity and edema. NME lesions were seen as asymmetric densities in seven patients on mammography.

On the T2-weighted images, all of the lesions were hyperintense in comparison to the surrounding breast tissue. The sizes of the lesions, as defined by the longest dimension on MRI, ranged from $0.7 \mathrm{~cm}$ to $6.7 \mathrm{~cm}$ (median, $3.6 \mathrm{~cm}$ ). According to imaging findings, all patients were attributed to BI-RADS 4 and underwent Tru-cut biopsy for a definitive diagnosis.
Table 1. Magnetic Resonance Imaging Features in 20 Patients With IGM

\begin{tabular}{|c|c|}
\hline Characteristic & Number \\
\hline Total number of breasts involved with IGM & 20 \\
\hline Total number of lesions & 29 \\
\hline Mass lesions & 14 \\
\hline \multicolumn{2}{|l|}{ Shape } \\
\hline Round & 9 \\
\hline Oval & 5 \\
\hline \multicolumn{2}{|l|}{ Margin } \\
\hline Smooth & 11 \\
\hline Irregular or spiculated & 3 \\
\hline \multicolumn{2}{|l|}{ Mass enhancement } \\
\hline Homogeneous & 3 \\
\hline Heterogeneous & 1 \\
\hline Rim enhancement & 10 \\
\hline Non-mass enhancement & 15 \\
\hline \multicolumn{2}{|l|}{ Distribution } \\
\hline Segmental & 6 \\
\hline Regional & 4 \\
\hline Diffuse & 3 \\
\hline Linear & 2 \\
\hline \multicolumn{2}{|l|}{ Internal enhancement } \\
\hline Homogeneous & 3 \\
\hline Heterogeneous & 8 \\
\hline Clumped or confluent & 4 \\
\hline
\end{tabular}

Abbreviation: IGM: Idiopathic granulomatous mastitis.

\section{Discussion}

IGM is a rare chronic inflammatory disease of the breast characterized by granuloma and abscess formations. It was first defined by Milward and Gough (1) and subsequently by Kessler and Wolloch (2). It is generally seen in premenopausal women in their second to fourth decades, and mostly in the first six years after childbirth (3). Our study supported this age characterization; with one exception, all of our patients were under 50-years-old.

IGM patients may present clinically with complaints of unilateral or bilateral palpable masses. Masses that clinically suspected of being breast cancer may be accompanied by nipple retraction, skin thickening, and a fistula tract. Approximately $15 \%$ of cases may have axillary lymphadenopathy (5). Using MRI, we detected skin thickening in nine of our 20 cases, and noted breast edema and nipple retraction in five and nine cases, respectively. Five of our 

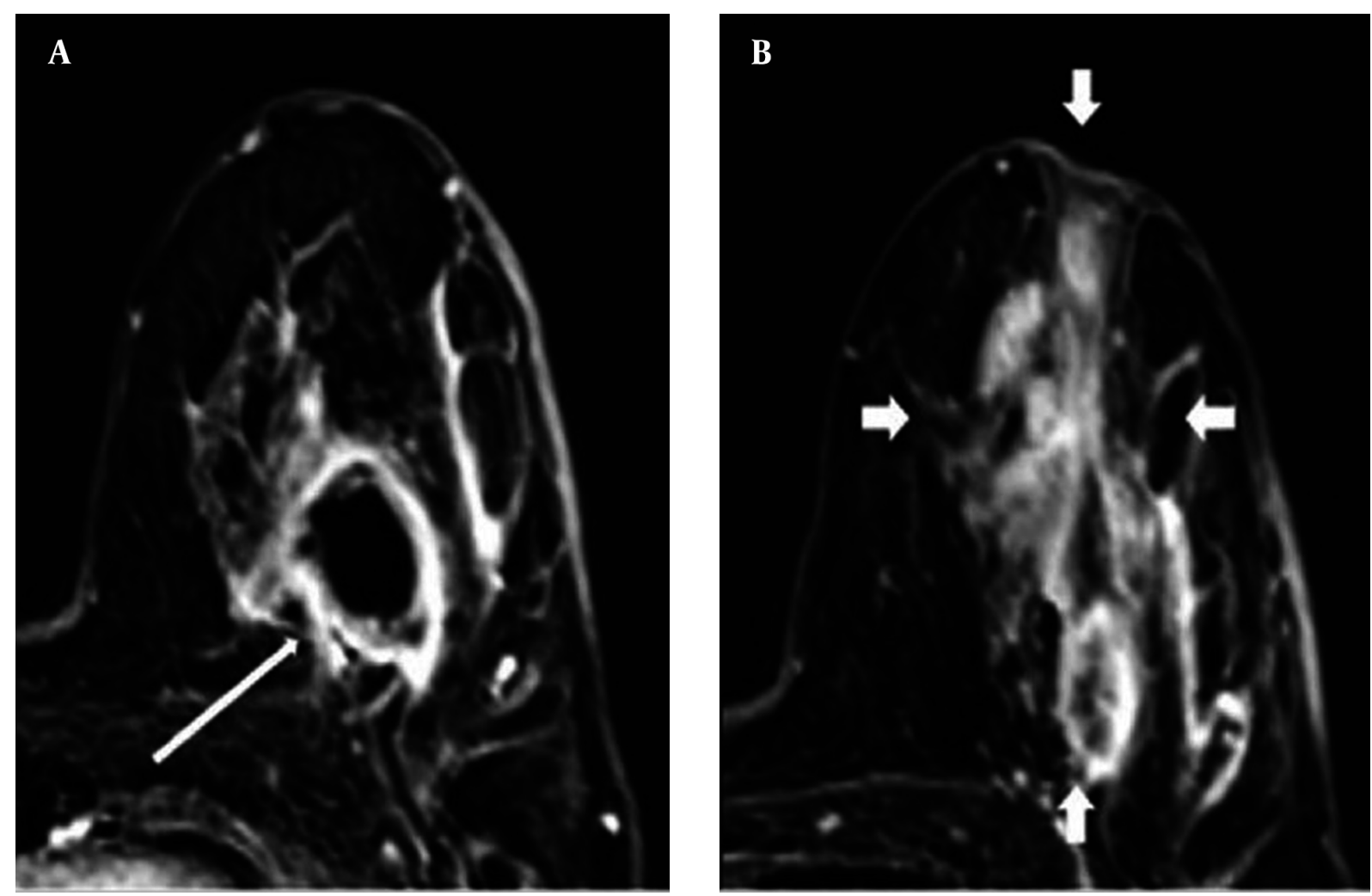

Figure 1. A 38-year-old woman with idiopathic granulomatous mastitis. A, Axial subtracted T1-weighted MR images demonstrated an ill-defined mass lesion with rim enhancement (thin arrow); B, A regional non-mass lesion with heterogeneous enhancement (thick arrows).

patients had axillary lymphadenopathy, and we saw fistula tracts in four patients.

The mammographic and ultrasonography findings of IGM have been reported in various studies. The mammographic findings can be completely normal; nevertheless, asymmetric densities or masses that mimic carcinoma can sometimes be determined even if no calcification is observed. A patient's inability to tolerate the appropriate amount of compression because of painful, sensitive breast tissue due to inflammation is a factor that complicates mammographic assessment. Ultrasonography frequently shows hypoechoic single or multiple masses with or without tubular connections. Sometimes, abscesses and fistula tracts can be detected in diffuse heterogeneous breast parenchyma (4, 6-10). Our results were similar to the mammographic and sonographic findings in the literature. We also found asymmetric densities, and circumscribed and ill-defined masses, on mammography. The corresponding ultrasonography for these patients detected various features, including heterogeneous hypoechoic lesions with or without tubular extension, parenchymal edema, and abscess formation.
Overall, breast MRI has high sensitivity, reaching 91\% $-100 \%$ in the detection of malignant lesions $(11,12)$. MRI is used in the preoperative staging of tumors, primary tumor assessments, protective breast treatment follow-up, and in women with a high risk of cancer. It is also used as a problem-solver in patients who cannot be diagnosed by mammography and ultrasonography (13). Breast MRI has been in routine clinical use for the last 20 years, and although it has significantly decreased the number of unnecessary biopsies and surgical interventions, its specificity has unfortunately remained at insufficient levels (37\% - 86\%) (14).

Various recently published studies on MRI findings in the diagnosis of IGM have reported that IGM demonstrates very unstable morphological and contrast-enhancement features on MRI. These MRI instabilities are in line with the histopathological findings of the disease at different stages, such as inflammatory reaction, abscess, and fibro$\operatorname{sis}(6,7)$.

Dursun et al. (6) evaluated the imaging features of IGM in a series of 36 cases and concluded that IGM mimics breast cancer. The authors found that mass lesions on MRI 

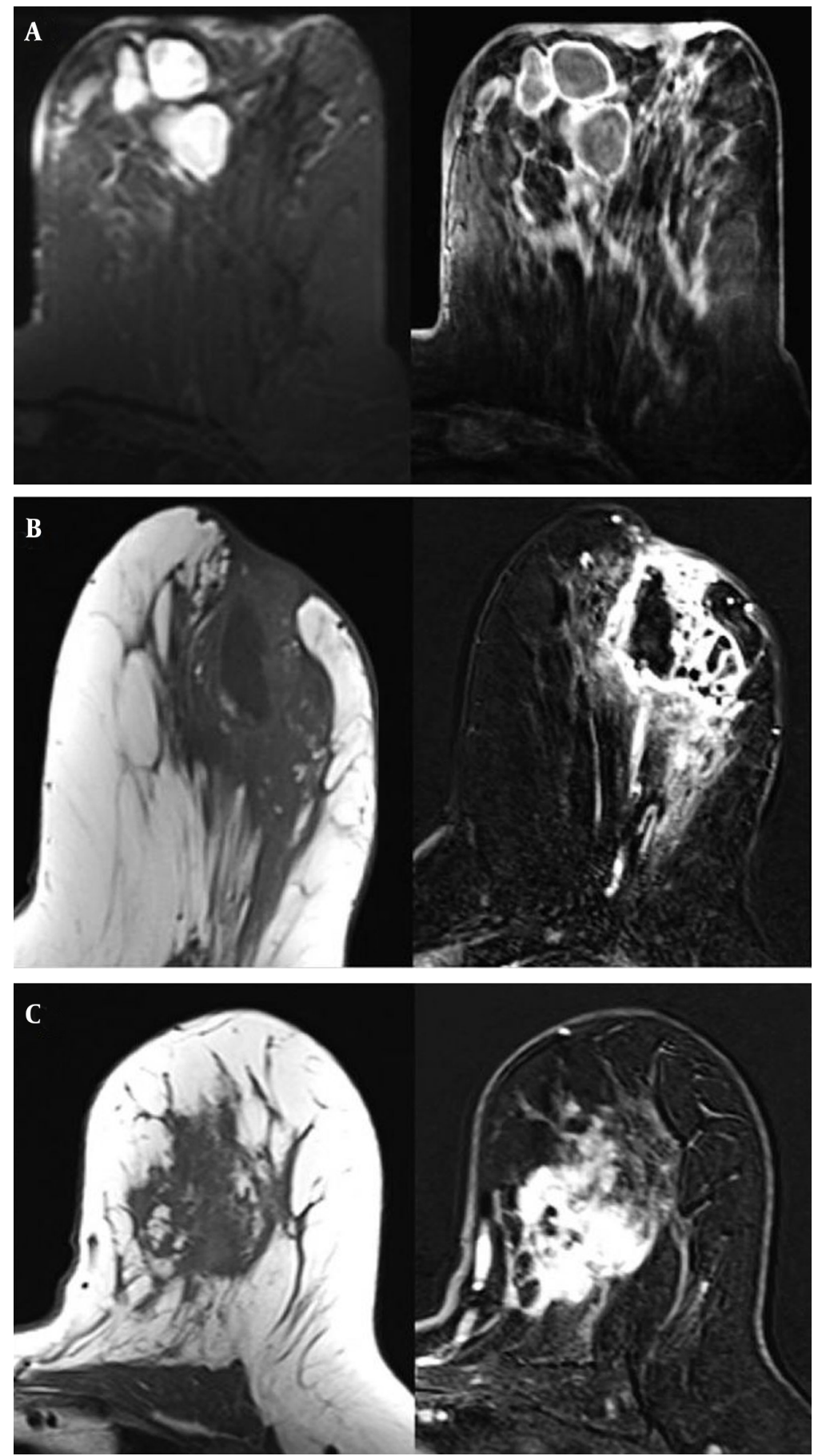

Figure 2. A-C. Axial fat-supressed T2 and subtracted T1-weighted MR images show multiple abscesses with rim enhancement and periareolar skin thickening (A). Precontrast axial $\mathrm{T} 1$ and postcontrast subtracted T1-weighted images show regional heterogeneous enhancement including necrotic areas, skin involvement and areolar retraction (B). A 39-year-old woman with idiopathic granulomatous mastitis, axial precontrast T1 and postcontrast subtracted images reveal a mass with irregular and heterogeneous enhancement (C).

most frequently had round shapes, smooth contours, and rim enhancement features. They also most commonly en- countered homogeneous or heterogeneous enhancement patterns that had segmental distributions in non-mass- 

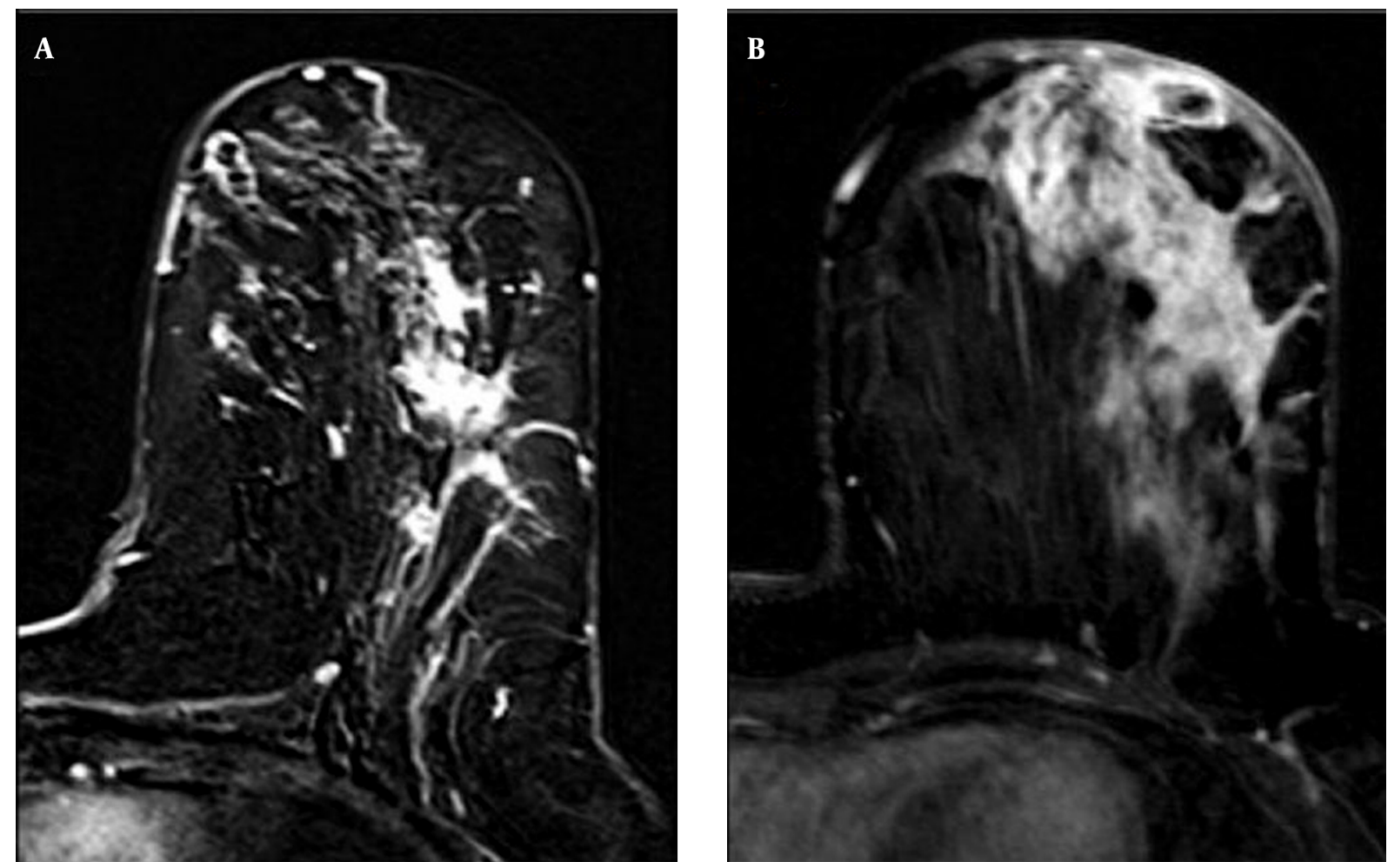

Figure 3. Axial postcontrast T1-weighted subtracted MR images of different patients demonstrate clumped (A) and segmental enhancement in non-mass lesions (B). Diffuse thickened skin could be seen (B).

like lesions. The most common findings of Kocaoglu et al. (7) were segmental heterogeneous enhancements and multiple ring-like abscess formations. Of particular note is that ring-like abscess formations have been the most frequently seen finding in other studies, as well (8).

In our study, the most common patterns in mass lesions on MRI were rounded shapes, smooth borders, and rim enhancements. The most characteristic finding of IGM in our series was also peripheral rim enhancement, indicating abscess formation. In spite of the other benign features (round, smooth, etc.), we included rim enhancement lesions on MRI in BI-RADS 4. Although various opinions have been offered in the literature, the rim enhancement feature is not considered a strong indicator of malignancy (15). We most frequently observed segmental distribution and heterogeneous enhancement patterns in the NME lesions in our study. Two patients had linear enhancement patterns. Segmental or linear enhancements have been reported as features of ductal carcinoma in situ on MRI (16).

Irregular masses, which could be confused with malignancy on MRI findings in IGM, have also been reported (5, 6). This feature is formed histopathologically due to noncaseified granulomas in the breast lobules. We found three such cases in our study. It has been reported that an irregular structure has the highest positive predictive value for a malignant lesion (17). Thus, this is one of the features of IGM that is most often confused with breast carcinoma. Some patients may have sinus tracts on MRI, but we observed those in only two of the patients in our series. Ultrasonography, similar to MRI, was superior to mammography in detecting mass lesions in IGM patients. The number of NME lesions on ultrasonography was lower than that seen on MRI. In accordance with the literature, mammography was limited in the evaluation of these patients compared with MRI and ultrasonography in our study, due to poor patient tolerance for breast compression in IGM that can make it difficult to obtain high-quality mammography. On the other hand, MRI showed greater accuracy for indicating the spread of lesions in our patients, compared to mammography and ultrasonography. Unfortunately, however, these findings were non-specific and therefore not sufficiently adequate to differentiate IGM from breast carcinoma.

Dursun et al. (6) found the time-signal intensity curve to be benign for IGM, and stated that this could contribute to the differential diagnosis of malignancy. In our study, 

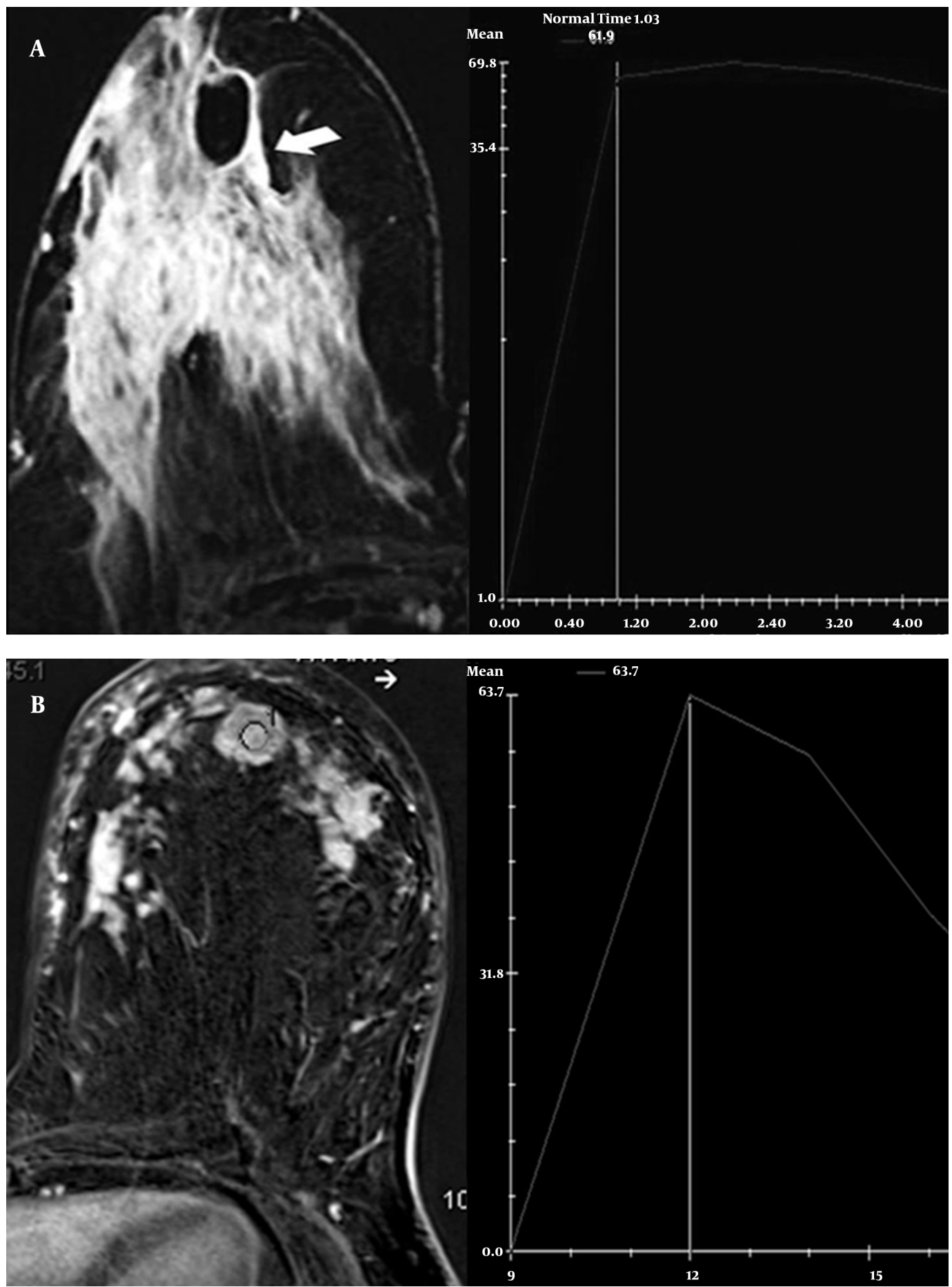

Figure 4. Axial postcontrast T1-weighted subtracted MR image demonstrates a regional non-mass enhancement including rim enhancement pattern. A, The time-signal intensity curve shows initial increasing and plateau. B, MRI of another patient shows homogeneous mass enhancement in the left breast. The time-signal intensity curve demonstrates initial increasing and wash-out pattern like a carcinoma.

the kinetic curve evaluation for IGM was generally found

to be a benign persistent type 1 time-signal intensity curve, 
but malignant washout type 3 was also detected.

To our knowledge, with 20 cases, our series is currently the second-most extensive study in the literature to evaluate the MRI features of IGM. Nevertheless, more studies are needed to define the MRI features of IGM. Furthermore, one of the most significant limitations of our study was that the variability of the observers was not taken into consideration. Studies in the literature have also reported changes among observers in the assessment of lesions in breast MRI (18).

The radiologic findings of IGM have a wide spectrum on MRI. Although no radiographic findings are specific for IGM, rim enhancement lesions on MRI should be strongly considered for this entity. Time-signal intensity curve analyses can provide useful information in distinguishing IGM from malignant breast lesions. The findings of our study suggest that breast MRI cannot be used definitively to differentiate breast carcinoma from IGM.

Although MRI can aid mammography and ultrasonography in the classification of lesions according to the BIRADS lexicon in some cases, it occasionally causes confusion and thus increases the number of unnecessary biopsies and procedures. Histopathological assessment is still the most accurate method for the diagnosis of IGM.

In this study, we present the MRI imaging features of a series of 20 cases with histologically proven IGM. Our results will help radiologists understand the atypical imaging findings of IGM.

\section{Acknowledgments}

We thank Necmettin Erbakan University of Medical Sciences for their valuable support.

\section{References}

1. Milward TM, Gough MH. Granulomatous lesions in the breast presenting as carcinoma. Surg Gynecol Obstet. 1970;130(3):478-82. [PubMed: 5461013].

2. Kessler E, Wolloch Y. Granulomatous mastitis: a lesion clinically simulating carcinoma. Am J Clin Pathol. 1972;58(6):642-6. [PubMed: 4674439].

3. Jorgensen MB, Nielsen DM. Diagnosis and treatment of granulomatous mastitis. Am J Med. 1992;93(1):97-101. [PubMed: 1626579].

4. American College of Radiology. . Breast Imaging Reporting and Data System. 4th ed. Reston VA, editor. USA: Elsevier; 2003.
5. Cakir B, Tuncbilek N, Karakas HM, Unlu E, Ozyilmaz F. Granulomatous mastitis mimicking breast carcinoma. Breast J. 2002;8(4):251-2. [PubMed: 12100120].

6. Dursun M, Yilmaz S, Yahyayev A, Salmaslioglu A, Yavuz E, Igci A, et al. Multimodality imaging features of idiopathic granulomatous mastitis: outcome of 12 years of experience. Radiol Med. 2012;117(4):529-38. doi: 10.1007/s11547-011-0733-2. [PubMed: 22020426].

7. Kocaoglu M, Somuncu I, Ors F, Bulakbasi N, Tayfun C, Ilkbahar S. Imaging findings in idiopathic granulomatous mastitis. A review with emphasis on magnetic resonance imaging. J Comput Assist Tomogr. 2004;28(5):635-41. [PubMed: 15480037].

8. Al-Khawari HA, Al-Manfouhi HA, Madda JP, Kovacs A, Sheikh M, Roberts O. Radiologic features of granulomatous mastitis. Breast J. 2011;17(6):645-50. doi: 10.1111/j.1524-4741.2011.01154.x. [PubMed: 21929558].

9. Ozturk M, Mavili E, Kahriman G, Akcan AC, Ozturk F. Granulomatous mastitis: radiological findings. Acta Radiol. 2007;48(2):150-5. doi: 10.1080/02841850601128975. [PubMed: 17354134].

10. Han BK, Choe YH, Park JM, Moon WK, Ko YH, Yang JH, et al. Granulomatous mastitis: mammographic and sonographic appearances. AJR Am J Roentgenol. 1999;173(2):317-20. doi: 10.2214/ajr.173.2.10430126. [PubMed: 10430126].

11. Orel SG, Schnall MD, LiVolsi VA, Troupin RH. Suspicious breast lesions: MR imaging with radiologic-pathologic correlation. Radiology. 1994;190(2):485-93. doi: 10.1148/radiology.190.2.8284404. [PubMed: 8284404].

12. Bone B, Pentek Z, Perbeck L, Veress B. Diagnostic accuracy of mammography and contrast-enhanced MR imaging in 238 histologically verified breast lesions. Acta Radiol. 1997;38(4 Pt 1):489-96. [PubMed: 9240665].

13. Sardanelli F, Boetes C, Borisch B, Decker T, Federico M, Gilbert FJ, et al Magnetic resonance imaging of the breast: recommendations from the EUSOMA working group. Eur J Cancer. 2010;46(8):1296-316. doi: 10.1016/j.ejca.2010.02.015. [PubMed: 20304629].

14. Kriege M, Brekelmans CT, Boetes C, Besnard PE, Zonderland HM, Obdeijn IM, et al. Efficacy of MRI and mammography for breast-cancer screening in women with a familial or genetic predisposition. $N$ Engl J Med. 2004;351(5):427-37. doi: 10.1056/NEJMoa031759. [PubMed: 15282350].

15. Liberman L, Morris EA, Lee MJ, Kaplan JB, LaTrenta LR, Menell JH, et al. Breast lesions detected on MR imaging: features and positive predictive value. AJR Am J Roentgenol. 2002;179(1):171-8. doi: 10.2214/ajr.179.1.1790171. [PubMed:12076929].

16. Mossa-Basha M, Fundaro GM, Shah BA, Ali S, Pantelic MV. Ductal carcinoma in situ of the breast: MR imaging findings with histopathologic correlation. Radiographics. 2010;30(6):1673-87. doi: 10.1148/rg.306105510. [PubMed: 21071382].

17. Tozaki M, Igarashi T, Fukuda K. Positive and negative predictive values of BI-RADS-MRI descriptors for focal breast masses. Magn Reson Med Sci. 2006;5(1):7-15. [PubMed: 16785722].

18. Beresford MJ, Padhani AR, Taylor NJ, Ah-See ML, Stirling JJ, Makris $A$, et al. Inter- and intraobserver variability in the evaluation of dynamic breast cancer MRI. J Magn Reson Imaging. 2006;24(6):1316-25. doi: 10.1002/jmri.20768. [PubMed: 17058203]. 